? Byetta $^{\varpi}$ und Bydureon ${ }^{\oplus}$, wie das neue Präparat voraussichtlich heißen wird, sollen parallel auf dem Markt existieren. Für wen würden Sie was empfehlen? Forst: Generell halte ich die einmal wöchentliche Darreichung für die potentere. Allerdings hat die zweimal tägliche Galenik den Vorteil der besseren postprandialen Wirkung, was sie für Patient mit besonders starkem Glukoseanstieg nach dem Essen prädestinieren könnte. Zudem ist die Zulassung für Byetta $^{\circledast}$ in Kombination mit Basalinsulin bereits eingereicht, für die neue Form wird es wohl noch zwei Jahre bis zu dieser Indikationserweiterung dauern.

\section{Alternative bei Typ-2-Diabetes Gliptin steht Glitazon in der Wirkung nicht nach}

- Bei unbehandeltem Typ-2-Diabetes haben Sitagliptin und Pioglitazon im direkten Vergleich die Blutzuckerkontrolle gleichermaßen klinisch signifikant verbessert. Der DPP-4-Hemmer Sitaglitptin mit dem derzeit breitesten Zulassungsspektrum (als Januvia ${ }^{\circledR}$ und Xelevia ${ }^{\otimes}$ oder kombiniert mit Metformin als Janumet ${ }^{\circledR}$ und Velmetia ${ }^{\circledR}$ im Handel) bietet sich daher als Alternative an, wenn das Glitazon nun nicht mehr uneingeschränkt zulasten der GKV verordnet werden kann.

An der randomisierten Studie nahmen 492 Typ2-Diabetiker mit moderater bis schwerer Hyperglykämie teil. Nach der ersten Doppelblindphase über 12 Wochen unter Monotherapie (Sitagliptin $100 \mathrm{mg} / \mathrm{d}$, Pioglitazon $15 \mathrm{mg} / \mathrm{d}$, nach sechs Wochen hochtitriert auf $30 \mathrm{mg} / \mathrm{d}$ ) war der $\mathrm{HbA}_{1 \mathrm{c}}$-Wert in beiden Gruppen ähnlich gesunken (um $-1 \%$ bzw. $-0,9 \%$ ). In einer zweiten Phase von 28 Wochen wurde in der Sitagliptin-Gruppe dieses durch die Fixkombination Sitagliptin/Metformin (Sita/Met) ersetzt, in der Vergleichsgruppe Pioglitazon auf $45 \mathrm{mg} /$ Tag hochtitriert. Am Ende dieser Phase war der Therapieerfolg mit Sita/Met signifikant stärker. Der $\mathrm{HbA}_{1 \mathrm{c}}$ war um 1,8\% (vs. 1,4\%) gesunken, 55\% der Patienten (vs. 40,5\% mit Pioglitazon) lagen unter dem $\mathrm{HbA}_{1 \mathrm{c}}$-Ziel von $7 \%$.

Die Verträglichkeit war in beiden Behandlungsgruppen durchweg gut. Hypoglykämie-Episoden spielten keine wesentliche Rolle (Inzidenz 2,3\% bzw. 2.2\%). Mit Sita/Met traten signifikant weniger Ödeme auf (0,9 vs. $6,1 \%)$, mit Pioglitazon seltener gastrointestinale Nebenwirkungen. Als wesentlicher Vorteil der Sita/Met-Therapie erwies sich die Gewichtsabnahme von im Mittel 1,1 kg, während die Patienten mit Pioglitazon im Mittel 3,4 kg zulegten.
? Gibt es ein spezielles Gerät zur Applikation der neuen Darreichungsform?

Forst: Nein, die Injektionslösung wird aus einem Pulver und einer Flüssigkeit gemischt dann über eine Spritze appliziert. Dabei ist die Nadel wegen der Mikrosphären messtechnisch etwa ein Drittel dicker als eine Nadel für Insulinpens. Gefühlstechnisch spürt der Patient beim Einstich aber keinen Unterschied zu einer Insulininjektion.

Interview: Sarah Louise Pampel

1. Best JH, Hoogwerf BJ, Herman WH et al. Diabetes Care 2011;34(1):90-5.

\section{Kurz notiert}

\section{Neuer GLP-1-Agonist: Direktvergleich in Phase-III}

Nachdem sich der GLP-1-Rezeptoragonist Lixisenatid in einer placebokontrollierten Phase-III-Studie bei Typ-2-Diabetes bereits als gut wirksam und sicher erwiesen hatte, war er nun auch im Direktvergleich mit Exenatide erfolgreich. In der GetGoal-X-Studie erwies sich das einmal täglich zu injizierende Lixisenatid dem zweimal täglich zu injizierenden Konkurrenzprodukt bei der $\mathrm{HbA}_{1 c}$-Senkung als nicht unterlegen (primärer Endpunkt). Zudem wurden mit dem neuen GLP-1-Agonisten signifikant weniger Hypoglykämien verzeichnet als mit dem Vergleichspräparat (symptomatische: $2,5 \%$ versus 7,9\%), wie das Herstellerunternehmen Sanofi Aventis mitgeteilt hat.

GetGoal X ist eine offene randomisierte Multicenterstudie mit 639 Typ-2Diabetikern über 24 Wochen, die mit Metformin unzureichend eingestellt waren. Die beiden Inkretinmimetika wurden zusätzlich zu Metformin in einer Dosis von bis zu 20 Mikrogramm pro Tag injiziert. Die Studienergebnisse sollen im Detail demnächt bei einem Fachkongress vorgestellt werden.

\section{Blutzucker einfach messen und dokumentieren}

Die Kombination aus Blutzucker-(BZ-)messgerät und integrierter Diabetesmanagement-Software im Contour ${ }^{\circledR}$ USB bietet für Anwender wie Betreuer Vorteile. Das ist das Ergebnis einer sechswöchigen Beobachtungsstudie unter der Leitung des Hamburger Diabetologen Prof. Ulrich Schwedes, an der sich deutschlandweit 1127 insulintherapierte Typ-1- und Typ-2 Diabetiker beteiligten. Nur ein Drittel der Teilnehmer gab an, bereits zuvor seine Blutzuckerdaten verwaltet zu haben. Fast alle Patienten (97,3\%) äußerten sich zufrieden und wollen das System weiterhin verwenden. Besonders positiv wurden die automatische Codierung, der Speicher von bis zu 2000 Werten, die USB-Schnittstelle und das einfache Dokumentieren und Auswerten bewertet.

Uneingeschränkt positiv ist nach Angaben des Unternehmens Bayer Vital auch die Meinung der beteiligten Ärzte und Diabetesberater. Alle beurteilten die Handhabung des Geräts als mindestens gut. Sie bestätigten, dass es zu einer besseren Compliance $(69,5 \%)$ sowie leichteren Identifizierung von BZ-Entgleisungen $(58,6 \%)$ und so zu einer besseren Kontrolle der BZ-Einstellung (71,9\%) beiträgt. In einer Sub-Analyse waren zudem Nüchtern-BZ, postprandialer $\mathrm{BZ}$ und $\mathrm{HbA}_{1 \mathrm{c}}$ nach dem Abschlussbesuch niedriger als die Ausgangs-Werte. 